\title{
Menschen mit geistiger Behinderung und Alkoholproblemen im Spiegel der Suchthilfe
}

\author{
M. Schubert
}

\author{
People with Mental Retardation and Alcohol Problems in View of \\ Treatment Programs
}

\section{Zusammenfassung}

In einem Forschungsprojekt wurde die bislang weithin unbeachtete Thematik Sucht bei Menschen mit geistiger Behinderung näher untersucht. Hierzu wurden Suchthilfeeinrichtungen in SachsenAnhalt zu Erfahrungen mit Betroffenen mit Lernschwierigkeiten mittels Fragebogen schriftlich befragt. Die Ergebnisse weisen nach, dass übermäßiger Konsum auch in dieser Subpopulation als relevantes Problem einzuschätzen ist. Wenngleich die Mitarbeiter versuchen, ihre Angebote an die spezifischen Voraussetzungen der Personengruppe anzupassen, zeigt sich auch, dass weithin tragfähige Konzepte zur Behandlung im ambulanten und stationären Bereich fehlen. Vor diesem Hintergrund wird die Notwendigkeit der Konzeptentwicklung unterstrichen und für eine verstärkte Zusammenarbeit von Sucht- und Behindertenhilfe plädiert, um eine effektive Kooperation im Sinne der Betroffenen zu fördern.

\section{Schliuisselwörter}

Geistige Behinderung · Behandlung · Alkohol

\section{Abstract}

During a research project we focused on alcohol (ab)use among people with mental retardation. Using a questionnaire about experiences with this subpopulation we surveyed institutions providing drug treatment programs in Saxony-Anhalt. Our findings prove that alcohol abuse has to be considered a relevant problem of mentally retarded people. Even though treatment programs try to cope with the specific needs of these clients, our findings suggest a lack of sustainable treatment conceptions for people with mental retardation. We underline therefore at first the need to develop special treatment conception and second a narrower and more effective cooperation between institutions for people with disabilities and drug treatment programs.

Key words

Mental retardation $\cdot$ treatment $\cdot$ alcohol

\section{Einleitende Bemerkungen}

Der Konsum von Suchtmitteln ist in unserer Gesellschaft weit verbreitet und in Bezug auf legale Stoffe, wie Alkohol und Tabak, weithin akzeptiert. So weist die Deutsche Hauptstelle für Suchtfragen für 2002 einen Pro-Kopf-Konsum an reinem Alkohol von 10,4 Litern aus und rechnet mit 7,8 Millionen Menschen im erwerbsfähigen Alter, die einen riskanten Alkoholkonsum aufweisen [1].
Wenngleich gesellschaftlich vielfältige Hilfs- und Behandlungsmöglichkeiten für Betroffene zur Verfügung gestellt werden, scheint für Menschen mit Lernschwierigkeiten im weiteren und Personen mit geistiger Behinderung ${ }^{1}$ im engeren Sinne aufgrund ihrer spezifischen individuellen Voraussetzungen die Partizipation an diesen Möglichkeiten zumindest deutlich erschwert zu sein. Betrachten wir diese Gruppe näher, lassen sich zunächst zwei Aspekte festhalten: Zum einen sind diese Menschen Merkmalsträger im

Institutsangaben

Medizinische Fakultät, Institut für Gesundheits- und Pflegewissenschaften, Martin-Luther-Universität Halle-Wittenberg, Halle/Saale

Korrespondenzadresse

Michael Schubert · Martin-Luther-Universität Halle-Wittenberg, Medizinische Fakultät, Institut für Gesundheits- und Pflegewissenschaften · Magdeburger Str. 8 - 06097 Halle/Saale .

E-mail: michael.schubert@medizin.uni-halle.de

Bibliografie

Suchttherapie 2006; 7: 24-28 @ Georg Thieme Verlag KG Stuttgart · New York

DOI 10.1055/s-2006-926539

ISSN 1439-9903 
doppelter Weise (geistige Behinderung und Suchtproblematik) und somit in der zahlenmäßigen Repräsentanz im Verhältnis zur Gesamtbevölkerung doppelt selten. Zum anderen gleichen sich im Zuge einer modernen Heilpädagogik die Wohn- und Lebensverhältnisse von Menschen mit geistiger Behinderung an die der Allgemeinbevölkerung an. Somit scheinen die Risiken und Gefährdungen von (missbräuchlichem) Substanzkonsum durch den Auszug aus (überbehütenden) Großeinrichtungen in gemeindeintegrierte Wohnformen für diese Personengruppe ebenso hoch wie in der Allgemeinbevölkerung. Die Frage in wie weit sich die Charakteristika dieser Personengruppe in Form einer erhöhten oder verringerten Vulnerabilität für Substanzkonsum auswirken ist wissenschaftlich umstritten [4-6]. Betrachtet man die bisherigen Erkenntnisse aus dem deutschen und angloamerikanischen Sprachraum, konzentriert sich der Suchtmittelkonsum bei unserer Zielgruppe auf Alkohol und Tabak. Diese sind legal, gut erreichbar, relativ billig und werden zudem extensiv beworben [7]. Aufgrund der meist deutlich schwerwiegenderen Folgen als beim Tabakkonsum konzentrierte sich unsere Untersuchung auf Alkohol.

Ausgangspunkt unserer Studie war der mehrfach vorgetragene Eindruck von Mitarbeitern aus der Praxis der Behindertenarbeit, dass Alkoholprobleme in dieser Zielgruppe in den letzten Jahren an Relevanz gewonnen hätten. Die Aufarbeitung der Forschungslage zeigt, dass bis auf wenige meist epidemiologische Untersuchungen im angloamerikanischen Sprachraum [8 - 18] die Thematik als weithin unerforscht gelten kann. Wenn bisherige Ergebnisse zeigen, dass Menschen mit geistiger Behinderung andere individuelle Voraussetzungen haben bzw. mitbringen, die in der Therapie (i. w. S.) „eigene“ Settings benötigen [19], weist dies die Notwendigkeit nach, sich mit dem Personenkreis wissenschaftlich auseinander zu setzen. Ein Teil der Ergebnisse unseres Forschungsprojektes, der sich mit den Erfahrungen und Einschätzungen der Einrichtungen der Suchthilfe mit Menschen mit geistiger Behinderung beschäftigt, wird im Folgenden vorgestellt.

\section{Methodik}

Bei der vorliegenden Studie handelt es sich um eine einrichtungsbezogene Vollerhebung im Land Sachsen-Anhalt. Befragt wurden alle Einrichtungen der Suchtkrankenhilfe sowie alle Wohneinrichtungen und Werkstätten für Menschen mit geistiger Behinderung in Bezug auf Alkoholkonsum und Umgang mit Alkoholproblemen bei Menschen mit geistiger Behinderung. Die Datenerhebung fand durch schriftliche Befragung mittels verschiedener Fragebögen statt, welche für diesen Zweck speziell entwickelt wurden. Der Fragebogen für Einrichtungen der Suchthilfe diente insbesondere zur Gewinnung von Informationen über zielgruppenbezogene Hilfs- und Behandlungsangebote. Aus Platzgründen beschränken wir uns hier großteils auf die Darstellung dieser Ergebnisse. Die differenzierten Resultate der Behindertenhilfe finden sich bei

\footnotetext{
1 Unter Personen mit einer Lernbehinderung wird ein IQ-Bereich von IQ 80/75-60/55 und für Menschen mit geistiger Behinderung ein Wert von IQ 60/55 und darunter verstanden. Dabei ist zu berücksichtigen, dass bei einer IQ-bezogene Sichtweise nur negative Merkmalsbeschreibungen (Defizite) in den Blick genommen werden. Insbesondere ist auch die Gleichsetzung von Intelligenz und Geist fraglich, so dass die Zahlen an sich nicht hinreichend diese Personengruppe charakterisieren [2, 3].
}

Schubert/Theunissen [20]. Die zum Teil umfangreichen Fragebögen umfassten insgesamt eine breite Themenpalette, um einerseits allgemeine und andererseits auch spezifische Daten zum Themenkreis Alkoholkonsum von Menschen mit geistiger Behinderung zu ermitteln. Alle Daten wurden über die Mitarbeiter der Einrichtungen bzw. der ambulanten Dienste gewonnen. Die Informationen wurden überwiegend mittels Rating-Skalen erhoben oder - wenn möglich - direkt erfragt. Die Auswertung der Daten erfolgte mittels des Statistikprogramms SPSS.

Von 118 ambulanten und stationären Einrichtungen der Suchthilfe zum Erhebungszeitpunkt nahmen trotz Vorinformations- und Erinnerungsschreiben nur 25 Institutionen an der Erhebung teil, was einem Rücklauf von $21 \%$ entspricht.

\section{Ergebnisse der Untersuchung}

Die 25 Institutionen unterteilen sich in 60\% Suchtberatungsstellen $(\mathrm{n}=15), 28 \%$ Fachkliniken ( $\mathrm{n}=7$ ), $8 \%$ Sozialpsychiatrische Dienste $(n=2)$ und $4 \%$ andere Einrichtungstypen $(n=1)$.

Die Einrichtungen (Tab.1) behandelten bzw. betreuten im Jahr 2002 insgesamt 5666 Personen. Von diesen waren laut Erstdiagnose 153 Personen (2,7\%) geistig behindert und $23(0,4 \%)$ wiesen eine Doppeldiagnose $^{2}$ auf. Zusätzlich zu den Daten bereits bestehender Diagnosen wurden die Mitarbeiter der Einrichtungen nach der Anzahl der Personen befragt, die nach Einschätzung des Ausfüllenden prä- oder komorbid eine geistige Behinderung aufwiesen. Mit 78 angegebenen Klienten/Patienten entspricht dies 1,4\%. Insgesamt kann daher von 254 Menschen mit geistiger Behinderung (4,5\%), die in Einrichtungen der Suchthilfe betreut/behandelt wurden, ausgegangen werden. Dieser Anteil (Abb.1) scheint dabei im Vergleich zu „vor einem Jahr“, „vor 3 Jahren“ und „vor 5 Jahren“ relativ konstant geblieben zu sein, auch wenn etwa ein Drittel der Institutionen keine Vergleiche zum Anteil von Menschen mit geistiger Behinderung in den vergangenen Jahren ziehen konnte.

Da für uns bei der Befragung insbesondere Erfahrungen und Probleme in der Betreuung bzw. Behandlung im Vordergrund standen, war einleitend zu klären, wie der Kontakt zu den Institutionen der Suchthilfe entsteht. Es zeigt sich, dass am häufigsten die Kontaktaufnahme über die Eltern bzw. die gesetzlichen Betreuer stattfindet (37\%). Bei 22\% der Personen kommen Anfragen von Mitarbei-

Tab. 1 Erfasste Personen in Suchthilfeeinrichtungen

\begin{tabular}{|c|c|c|}
\hline & Anzahl & in $\%$ \\
\hline Anzahl der Einrichtungen & $\mathrm{n}=25$ & \\
\hline behandelte/betreute Personen (gesamt) & $n=5666$ & $100 \%$ \\
\hline Personen mit geistiger Behinderung (It. Erstdiagnose) & 153 & $2,7 \%$ \\
\hline Personen mit Doppeldiagnose & 23 & $0,4 \%$ \\
\hline $\begin{array}{l}\text { Personen, die nach Einschätzung des Ausfüllenden eine } \\
\text { geistige Behinderung aufweisen }\end{array}$ & 78 & $1,4 \%$ \\
\hline Patienten/Klienten mit geistiger Behinderung (gesamt) & 254 & $4,5 \%$ \\
\hline
\end{tabular}

2 Geistige Behinderung und psychische (Vor-)Erkrankung, die nicht die Suchtproblematik betrifft. 
tern der Einrichtungen der Behindertenhilfe und zu $15 \%$ wird mittels ärztlicher Einweisung durch den Hausarzt der Kontakt hergestellt. Die Häufigkeiten einer notärztlichen Einweisung (5\%) oder eines richterlichen Beschlusses (1\%), welche nur stationäre Einrichtungen betreffen können, sind demgegenüber sehr gering. In der Kategorie „andere“ sind neben anderen sozialen Diensten und anderen Suchthilfeinstitutionen auch allgemein das soziale Umfeld genannt.

Als zweiter Analyseschritt wurde erfragt, wie die Mitarbeiter der Suchtkrankenhilfe in ihrer Arbeit auf die speziellen Voraussetzungen von Menschen mit geistiger Behinderung reagieren (können). Die Ergebnisse (Abb. 2) zeigen sehr deutlich, dass die meisten Einrichtungen sich durch vereinfachte Sprache und Wissensvermittlung über Alkohol (je 87\%) sowie durch das Setzen kleinerer, schneller erreichbarer Ziele (83\%) der Personengruppe anpassen. Weiterhin beziehen etwa die Hälfte der Einrichtungen stärker das Umfeld in ihre Arbeit ein (52\%) bzw. verlängern die (übliche) Behandlungsdauer (48\%). Etwa ein Drittel arbeitet verstärkt verhaltenstherapeutisch oder setzt häufiger Einzel- statt Gruppentherapie ein. Je $26 \%$ verwenden ein individualisierteres Behandlungskonzept oder nutzen verstärkt Elemente der Psychoedukation (z.B. soziales Problemlösungstraining). Außerdem setzen 13\% häufiger gestaltungstherapeutische Arbeitsansätze ein. Nur eine Einrichtung (4\%) gab an, dass ihr keine Möglichkeiten zur Anpassung gängiger Modelle zur Verfügung stehen.

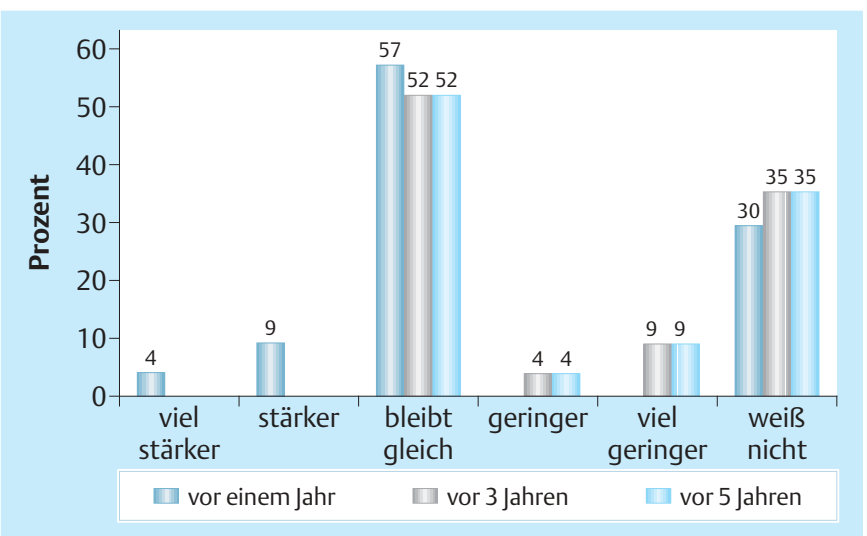

Abb. 1 Zeitliche Veränderung des Anteils geistig Behinderter in den Suchthilfeeinrichtungen $(n=23)$.
Daran anschließend stellt sich die Frage, welche Hindernisse in der Behandlung/Betreuung von Menschen mit geistiger Behinderung und Alkoholproblemen aus Sicht der Suchthilfe bestehen. Zunächst kann festgehalten werden, dass 36\% der Institutionen keine Schwierigkeiten mit dieser besonderen Zielgruppe haben. Auf der anderen Seite tragen die Probleme, die von den restlichen $64 \%$ der Einrichtungen gesehen werden, einen vielfältigen Charakter: Sie sind organisatorischer (z. B. Zeitaspekt, Personalprobleme), fachspezifischer (z. B. fehlende stationäre Angebote bzw. Anschlussheilbehandlungen, fehlende Konzepte) oder behinderungsspezifischer Art (z. B. Integrationsprobleme in die Behandlungsgruppe, fehlende stationäre Angebote bzw. Anschlussheilbehandlungen).

Aus dieser Fragestellung heraus lässt sich weiterführend die Frage ableiten, für wie geeignet sich die Einrichtungen für die Betreuung/Behandlung von Menschen mit geistiger Behinderung selbst halten (Abb.3). Dabei zeigt sich, dass die große Mehrheit (69\%) ihre Eignung eher schlechter (Kategorien 4-6) einschätzt. Andererseits sind geringere Werte an den (extremen) Enden der Skala zu finden, sodass die Eignung nur selten als „nicht“ oder „überhaupt nicht“ (je 13\%) eingeschätzt wird. Werden die Kategorien 3 und 4 zusammengefasst, so ergibt sich mit $60 \%$ eine klare „Tendenz zur Mitte“. Als zweiten Aspekt dieser Frage sollten die Einrichtungen Gründe für ihre Einschätzung angeben, bei der sowohl positive als auch negative Argumente angeführt wurden (Tab. 2).

Neben diesen isolierten Ergebnissen der Suchthilfe wurde die Schnittstelle zwischen Behinderten- und Suchthilfe, auf der sich die Betreuung und Behandlung von Menschen mit geistiger Behinderung und Suchtproblematik befindet, näher untersucht. In der vergleichend dargestellten Beurteilung (Abb.4) zur Qualität der Zusammenarbeit zeigen sich deutliche Parallelen in der (tendenziell positiven) Einschätzung. Andererseits fällt auf, dass in über der Hälfte der Institutionen der Behindertenhilfe kein Kontakt zur Suchthilfe besteht.

In Bezug auf Hilfs- und Unterstützungsmaßnahmen können aufgrund der Überschneidung der Hilfsanbieter bei Suchtproblemen von Menschen mit geistiger Behinderung Lücken auftreten. Daher beinhalteten die Bögen für beide Arten von Hilfsanbietern auch Items zum Aspekt der „Zuständigkeiten“. Zu bewerten waren hier-

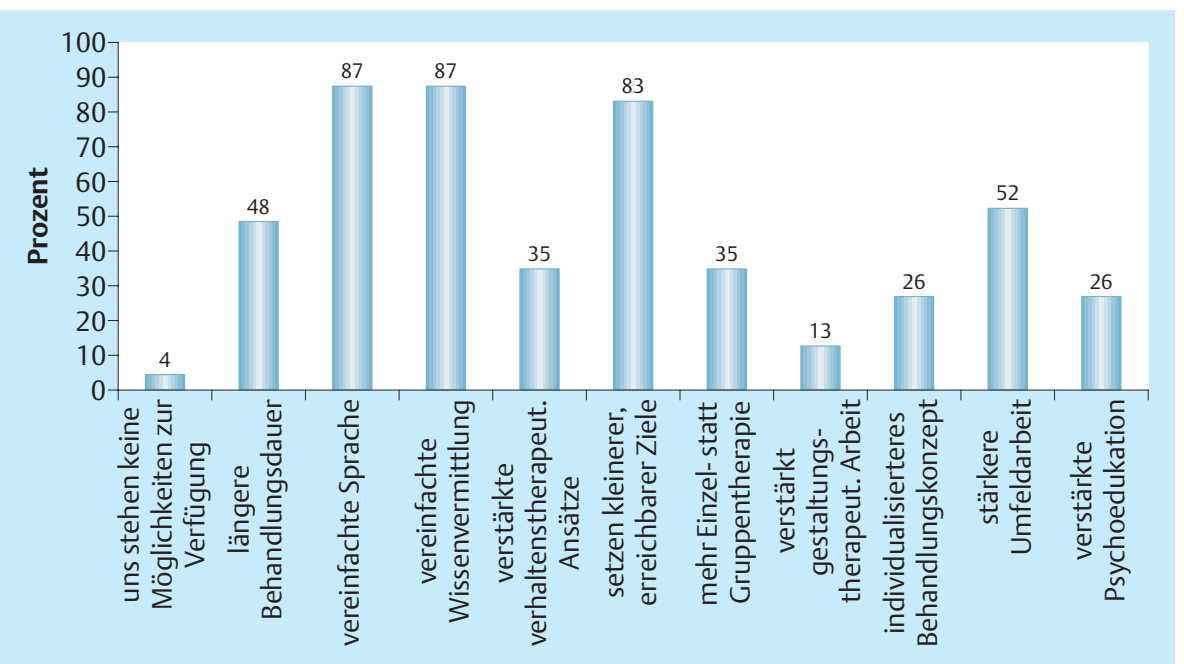

Abb. 2 Reaktionen der Suchthilfe auf die spez. Voraussetzungen von Menschen mit geistiger Behinderung und Suchtproblematik $(n=23)$. 


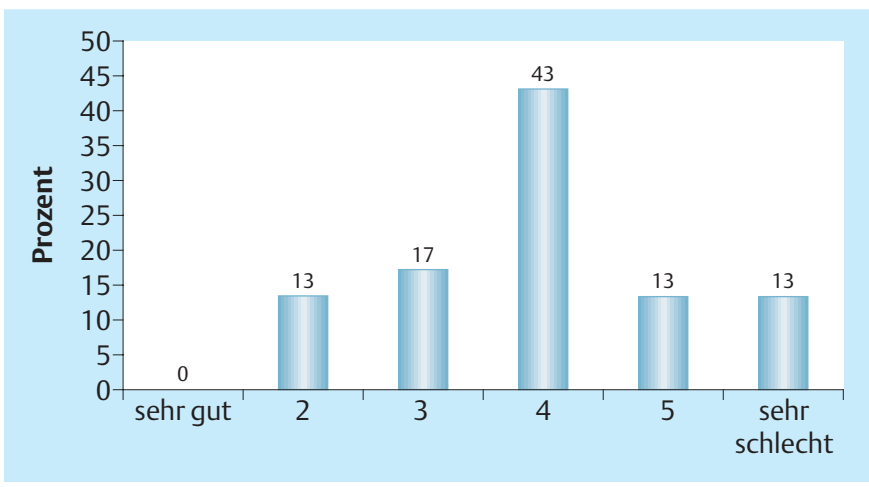

Abb. 3 Eignung der Einrichtung für Menschen mit geistiger Behinderung $(n=23)$.

Tab. 2 Gründe für die Einschätzung der Eignung für Menschen mit geistiger Behinderung

\begin{tabular}{|c|c|}
\hline negative Gründe & positive Gründe \\
\hline personelle Probleme $(5 x)$ & Arbeit speziell mit Menschen mit g. B. \\
\hline fehlende Qualifikation (2x) & individuelle Hilfen in der Betreuung \\
\hline räumliche Probleme $(2 \mathrm{x})$ & Familienarbeit \\
\hline $\begin{array}{l}\text { großer verbaler Anteil in der } \\
\text { Arbeit }\end{array}$ & Integration in die Sozio-R-Gruppe möglich \\
\hline kein einheitliches Konzept & $\begin{array}{l}\text { Beratung, Diagnostik, Hilfebedarfsermittlung } \\
\text { und Einleitung personenorientierter Hilfen } \\
\text { (z. B. ambulant betreutes Wohnen) }\end{array}$ \\
\hline Zeitmangel & Multiplikatoren \\
\hline keine ambulante Therapie & $\begin{array}{l}\text { Weiterbildung für die Behindertenhilfe in } \\
\text { Suchtfragen }\end{array}$ \\
\hline „Standardbehandlung“ & Kontakte zu relevanten Einrichtungen \\
\hline $\begin{array}{l}\text { konkrete Alltagsbegleitung } \\
\text { nicht umsetzbar }\end{array}$ & \\
\hline
\end{tabular}

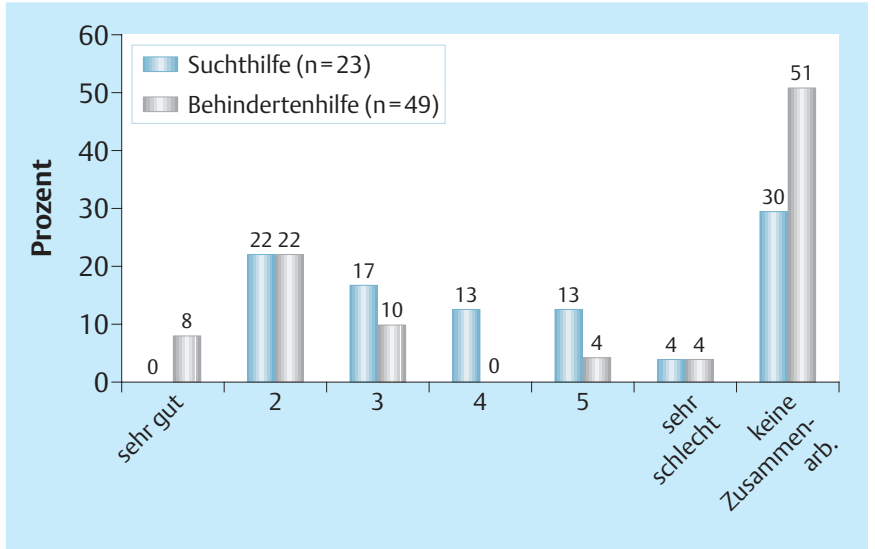

Abb. 4 Zusammenarbeit zwischen Sucht- und Behindertenhilfe.

bei zwei Aussagen, deren Antworten in den Abb. 5 und $\mathbf{6}$ dargestellt sind. Die Ergebnisse zeigen eine sehr deutliche starke Zustimmung sowohl der Behinderten- (64\% bzw. 74\%) als auch der Suchthilfe (je 64\%). Interessant ist dabei insbesondere das Ergebnis der zweiten Aussage (Abb. 6), da (über) zwei Drittel der Suchthilfeeinrichtungen die starke Notwendigkeit der Etablierung spezieller Angebote für Menschen mit Behinderung und Suchtproblematik sehen.

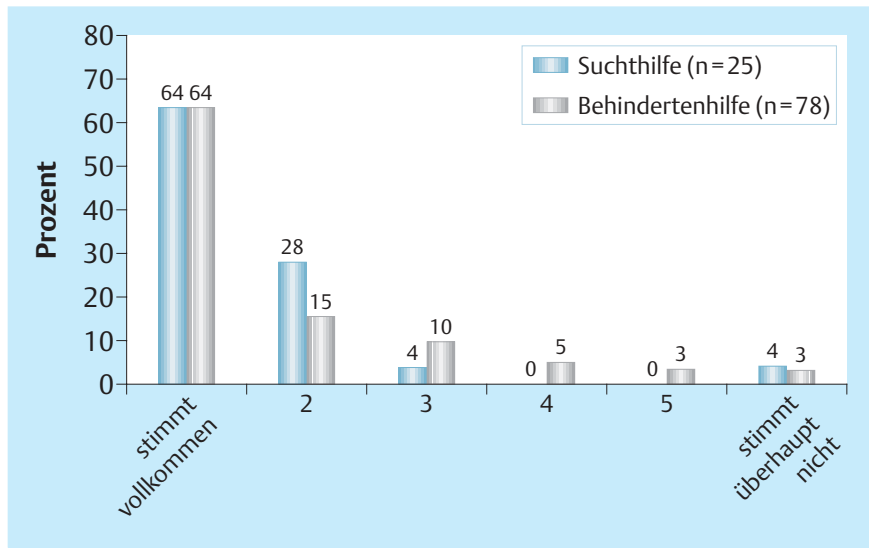

Abb. 5 Die Angebote der Suchthilfe müssen auch Menschen mit geistiger Behinderung zugänglich sein.

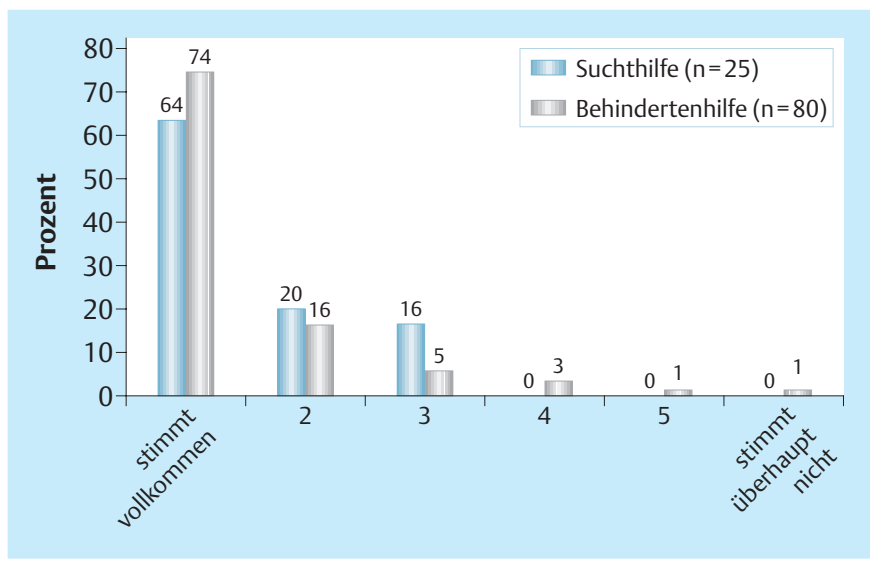

Abb. 6 Die Behindertenhilfe muss spezielle Angebote für suchtkranke Behinderte entwickeln.

\section{Diskussion}

Über unsere Erhebung in Bereich Sucht- und Behindertenhilfe konnten eine ganze Reihe neue, spezifische Informationen gewonnen werden. Diese sind insbesondere insofern zusätzlich von Wert, da bislang - speziell im deutschsprachigen Raum - nahezu nichts über Menschen mit geistiger Behinderung und Suchtproblematik bekannt ist. Dies gilt sowohl in Bezug auf Prävalenz und Konsumfolgen sowie auf Lebenswelt und Trinkmotive als auch auf den Bereich Therapie. Dabei bestimmt für die Suchthilfe insbesondere das Spannungsfeld zwischen sprachgebundenen Therapieformen und individuellen Voraussetzungen der Betroffenen die Problematik des Arbeitsfeldes.

Wenngleich bei unserer Untersuchung festgestellt werden kann, dass in den befragten Suchthilfeeinrichtungen „nur“ $4,5 \%$ der Klienten/Patienten Lernschwierigkeiten hatten, so entspricht dies jedoch immerhin der Prävalenz von 3\% einer geistigen Behinderung in der Allgemeinbevölkerung. Zudem weisen die Ergebnisse der Wohneinrichtungen der Behindertenhilfe (11\% Personen mit „riskantem Alkoholkonsum“) auf eine relevante Problematik hin. Andererseits stimmen Sucht- und Behindertenhilfe in ihrer Einschätzung überein, dass sich der Personenkreis von Menschen, die einen riskanten Alkoholkonsum zeigen, in den letzten Jahren nicht vergrößert zu haben scheint. Wenngleich ein (in den letzten Jahren 
häufig vollzogener) Umzug aus einer „beschützenden“ (Groß-)Einrichtung in selbstständigere, gemeindeintegrierte Wohnformen auch größere Risiken (wie eine Suchtproblematik) birgt, so zeigt die Konstanz des Phänomens, dass von einer direkten Gefährdung (z.B. durch die bessere Erreichbarkeit von Suchtmitteln) aufgrund der höheren Eigenverantwortung nicht ausgegangen werden kann, da sich die Anzahl der Betroffenen nicht signifikant erhöht hat [21].

Die Schwierigkeiten bei der Arbeit mit dieser Zielgruppe sind vielschichtig; neben organisatorischen Beschränkungen stehen dabei behinderungsspezifische Grenzen im Zentrum, welche die Integration in klassische Therapieprogramme erschweren. Treten zur Substanzproblematik weitere psychische Störungen oder Verhaltensauffälligkeiten hinzu, sind verstärkt individuelle und evtl. multiprofessionelle Arbeitsansätze gefragt. Sehr eng damit verbunden sind zudem die fachspezifischen Schwierigkeiten. Fehlende Konzepte und Erfahrungen für bzw. mit der Klientel zählen dazu ebenso wie fehlende stationäre und ambulante Angebote. Diese Befunde decken sich weithin auch mit Erfahrungen aus dem angloamerikanischen Sprachraum [6, 11, 15, 22, 23].

Betrachtet man die Antworten der Suchthilfeeinrichtungen zu inhaltlichen Fragen, lassen sich zwei verschiedene Tendenzen herausarbeiten. Zum einen sehen sich die Mitarbeiter der Suchteinrichtungen auch zuständig für Menschen mit Lernschwierigkeiten. Dies zeigen die zahlreichen Anpassungen der Therapie/Beratung an individuelle Voraussetzungen der Betroffenen, ebenso wie die sehr große Zustimmung zur Zugänglichkeit der Angebote für Menschen mit geistiger Behinderung. Zum anderen weist das Meinungsbild zur Frage, ob die Behindertenhilfe für geistig Behinderte spezielle Angebote entwickeln muss, auf eine eher entgegengesetzte Tendenz hin. Hier votierten Sucht- und Behindertenhilfe gleichermaßen deutlich für die Schaffung spezifischer Hilfs- bzw. Unterstützungsmaßnahmen. Dies hätten speziell offene, niedrigschwellige Angebote für Menschen mit Behinderung zu sein, die stärker an die Behindertenhilfe zu koppeln wären.

Insgesamt scheint auf Grundlage unserer Untersuchung auch die generelle Zusammenarbeit zwischen beiden Systemen ausbaufähig zu sein. Der niedrige Rücklauf unserer Befragung könnte darauf hindeuten, dass viele Einrichtungen nicht geantwortet haben, da die Thematik für diese scheinbar irrrelevant ist. Persönliche Erfahrungen, auch während der Erhebungsphase, bestätigen den Befund, dass sich die Einrichtungen der Behindertenhilfe häufig eine stärkere und umfangreichere Zusammenarbeit wünschen. Zudem zeigen die genannten positiven Aspekte zur Eignung von Suchthilfeeinrichtungen für Menschen mit Behinderung und Suchtproblematik (vgl. Tab. 2), dass - insbesondere bei intensiver Zusammenarbeit mit den entsprechenden Diensten der Behindertenhilfe - Suchthilfeeinrichtungen adäquate Angebote für Betroffene anbieten können.

Wenngleich Personen mit geistiger Behinderung und Suchtproblematik häufig die Ausnahme in den Einrichtungen der Suchthilfe darstellen, so sollte dieser Gruppe deutlich mehr Aufmerksamkeit als bisher zu Teil werden. Im Zuge der Normalisierung und Angleichung der Lebensumstände von Menschen mit geistiger Behinderung muss davon ausgegangen werden, dass sich auch der Anteil von Menschen mit Alkoholproblemen an den der Allgemeinbevölkerung annähert. Insbesondere die große konzeptio- nelle Lücke im Bereich Behandlung und Therapie dieser Personengruppe verweist auf die Notwendigkeit weiterer Forschung. Eine Grundlegung aus pädagogischer Sicht, wie bei Theunissen und Theunissen/Schubert [6, 24], kann dabei nur ein Anfang sein.

\section{Literatur}

${ }^{1}$ Deutsche Hauptstelle für Suchtfragen (DHS). Jahrbuch Sucht 2004 Geesthacht, 2003

${ }^{2}$ Speck O. Menschen mit geistiger Behinderung und ihre Erziehung: Ein heilpädagogisches Lehrbuch. München/Basel, 1999

${ }^{3}$ Theunissen G. Pädagogik bei geistiger Behinderung und Verhaltensauffälligkeiten. Bad Heilbrunn, 2000

${ }^{4}$ Lingg A, Theunissen G. Psychische Störungen und geistige Behinderung. Freiburg im Breisgau, 2000

${ }^{5}$ Beer O. Suchtmittelgebrauch bei Menschen mit so genannter geistiger Behinderung. Geistige Behinderung 2004; 3: 255-269

${ }^{6}$ Theunissen G. Alkoholgefährdungen und Suchtprobleme bei Menschen mit geistiger Behinderung. In: Wüllenweber E (Hrsg). Soziale Probleme von Menschen mit geistiger Behinderung. Stuttgart, 2004

${ }^{7}$ Delaney D, Poling A. Drug abuse among mentally retarded people: An overlooked problem? Journal of Alcohol and Drug Education 1990; 35: $48-54$

${ }^{8}$ Krishef CH, DiNitto DM. Alcohol abuse among mentally retarded individuals. Mental Retardation 1981; 19: 151 - 155

${ }^{9}$ Huang AM. The drinking behavior of the educable mentally retarded and the nonretarded students. Journal of Alcohol and Drug Education 1981; 26: $41-50$

${ }^{10}$ DiNitto DM, Krishef $\mathrm{CH}$. Drinking patterns of mentally retarded persons. Alcohol, Health and Research World 1983/1984; 8: 40-42

${ }^{11}$ Edgerton RB. Alcohol and drug use by mentally retarded adults. American Journal of Mental Deficiency 1986; 90: 602 - 609

${ }^{12}$ Westermeyer J, Kemp K, Nugent S. Substance disorder among persons with mild mental retardation. The American Journal of Addictions 1996; $5: 32-31$

${ }^{13}$ Lawrenson H, Lindsay WR, Walker P. The pattern of alcohol consumption within a sample of mentally handicapped people in Tayside. Mental handicap Research 1995; 8

${ }^{14}$ Gress IR, Boss MS. Substance abuse difference among students receiving special education school services. Child psychiatry and human development 1996; 4: 235-246

${ }^{15}$ McGillicuddy NB, Blane HT. Substance use in individuals with mental retardation. Addictive Behaviours 1999; 6: 869-878

${ }^{16}$ Burghard JF, Donohue B, Azrin NH et al. Prevalence and treatment of substance abuse in the mentally retarded population: An empirical review. Journal of Psychoactive Drugs 2000; 3: 293 - 298

17 Rimmer JH, Barddock D, Marks B. Health characteristics and behaviours of adults with mental retardation residing in three living arrangements. Research in Developmental Disabilities 1995; 16

${ }^{18}$ Clarke JJ, Wilson DN. Alcohol problems and intellectual disabilities. Journal of intellectual disability research 1999; 2: 135-139

${ }^{19}$ Longo LP. Alcohol Abuse in Persons with Developmental Disabilities. The Mental Health Aspects of Developmental Disabilities 1997; 4: $61-64$

${ }^{20}$ Schubert M, Theunissen G. Alkoholkonsum von Menschen mit geistiger Behinderung. Vierteljahresschrift für Heilpädagogik und ihre Nachbargebiete 2005; 4: $312-325$

${ }^{21}$ Schubert M. Alkoholmissbrauch und -abhängigkeit bei Menschen mit geistiger Behinderung - eine empirische Studie aus Sachsen-Anhalt. unveröff. Forschungsbericht Halle/Saale: Institut für Rehabilitationspädagogik, 2004

22 Christian L, Poling A. Drug abuse in persons with mental retardation: a review. American Journal on Mental Retardation 1997; 2: 126-136

${ }^{23}$ Lottmann TI. Access to generic substance abuse services for persons with mental retardation. Journal of alcohol and drug education 1993: 39: $41-55$.

${ }^{24}$ Theunissen G, Schubert M. Alkoholismus und Geistige Behinderung. Vorr. In: Klauß T (Hrsg). Psychologie und Geistige Behinderung. Heidelberg, 2006 